\title{
Facile Synthesis of Amphiphilic Fluorescent Phosphorus Dendron-Based Micelles as Antiproliferative Agents: First Investigations
}

\author{
Jieru Qiu ${ }^{[a, b, c]}$, Liang Chen ${ }^{[a, b, d]}$, Mengsi Zhan ${ }^{d}$, Régis Laurent ${ }^{[a, b]}$, Jérôme Bignon ${ }^{[e]}$, Serge \\ Mignani $^{*[f, g]}$, Xiangyang Shi ${ }^{*[d, g]}$, Anne-Marie Caminade ${ }^{[a, b]}$ and Jean-Pierre Majoral ${ }^{*[a, b]}$
}

[a] Laboratoire de Chimie de Coordination du CNRS, 205 Route de Narbonne, BP 44099, 31077 Toulouse Cedex 4, France

[b] LCC-CNRS, Université de Toulouse, CNRS, Toulouse, France

[c] Pharmaceutical Informatics Institute, College of Pharmaceutical Sciences, Zhejiang University, Hangzhou 310058, People's Republic of China

[d] College of Chemistry, Chemical Engineering and Biotechnology, Donghua University, Shanghai 201620, People's Republic of China

[e] Institut de Chimie des Substances Naturelles du CNRS, 91198 avenue de la Terrasse, Paris Gif-sur-Yvette Cedex, France

[f] Université Paris Descartes, PRES Sorbonne Paris Cité, CNRS UMR 860, Laboratoire de Chimie et de Biochimie Pharmacologiques et Toxicologique, 45, rue des Saints Peres, 75006, Paris, France

[g] CQM - Centro de Química da Madeira, MMRG, Universidade da Madeira, Campus da Penteada, 9020-105 Funchal, Portugal

*To whom correspondence should be addressed. E-mail: S. Mignani: serge.mignani@parisdescartes.fr, serge.mignani@staff.uma.pt; X. Shi: xshi@dhu.edu.cn and J-P. Majoral: majoral@lcc-toulouse.fr

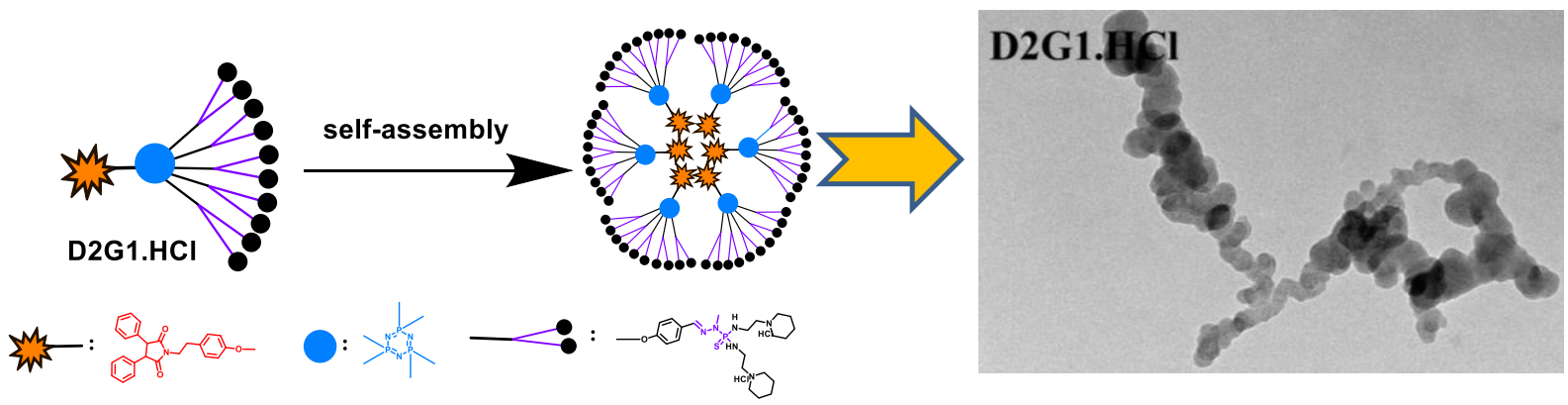

Abstract: We designed and synthetized several families of original amphiphilic fluorescent phosphorus dendron-based micelles showing relevant antiproliferative activities for use in the field of theranostic nanomedicine. Based on straightforward synthesis pathways, twelve amphiphilic phosphorus dendrons bearing 10 protonated cyclic amino groups (generation one), or 20 protonated amino groups (generation two), and one hydrophobic chain carrying one fluorophore moiety were created.. These original dendron-based micelles showed moderate to high antiproliferative activities against a panel of tumor cell lines. This paper presents for the first time the synthesis and our first investigations of original phosphorus dendron-based micelles for cancer therapy applications.

\section{Introduction}

In medicine, nanotechnologies have sparked rapidly growing interest as a result of their potential to revolutionize conventional therapeutic treatments. Over the past several decades, many engineered soft nanoparticles have been used, such as protein-drug conjugates, liposomes, polymer nanoparticles, polymer-lipid nanoparticles, hydrogel-nanoparticles composite system, dendrimers and dendrons. Further, inorganic nanoparticles have been developed for various uses, including to enhance the effectiveness of cancer treatments. ${ }^{1,2}$ Among the soft nanoparticles, dendrimers and dendrons, which are tree-like macromolecules that contain a central core, pertain to the "nano-world" by virtue of their size and degree of branching. Properties of dendrimers including nanometric size, multivalence, branch density, flexibility, and surface properties can be controlled, and provide versatility to the final functionalization and surface charge of the dendrimers, which is a feature that is distinct from uncontrollable polymer networks. Consequently, their remarkable properties enable 
them to play an increasingly important part in a diverse range of applications, including nanomedicine as components in drug-delivery applications that require host-guest interactions.

Soft nanoparticles facilitate drug-loading either via their interior or peripheral regions. ${ }^{3}$ For instance, polymeric micelles as micellar drug carriers are showing a high potential as nanomedicines to control the distribution of loaded bioactive molecules in the body as well as the biological activity based on adequate pharmacokinetic (PK) / pharmacodynamics (PD) behaviors. ${ }^{4}$ Several clinical applications of polymeric micelles for the treatment of cancer were described, ${ }^{5}$ such as the Phase II clinical trial of paclitaxel-loaded polymeric micelles against advanced pancreatic cancer. ${ }^{6}$ Nowadays, no polymeric micelle has been developed as drug active per se. An avenue recently explored in nanomedicine, involves the use of dendrimers and dendrons as active drugs. ${ }^{7}$ Numerous families of dendrimers exist as for example poly(amidoamine) (PAMAM) dendrimers, ${ }^{8}$ poly(propyleneimine) (PPI) dendrimers, ${ }^{9}$ poly(L-lysine) (PLL) dendrimers, ${ }^{10}$ poly(L-ornithine) (PLO) dendrimers, ${ }^{11}$ carbosilane dendrimers, ${ }^{12}$ phosphorus dendrimers. ${ }^{13}$ Of all types of dendrimers, ${ }^{14}$ phosphorus dendrimers and dendrons appear to be among one of the most useful dendritic systems as a result of the extraordinary reactivity and versatility of phosphorus chemistry. ${ }^{15}$ Through an assessment of properties of phosphorus dendrimers used in nanomedicine, we can highlight their key roles as drugs with activities against cancer, ${ }^{16}$ neurodegenerative diseases, ${ }^{17-23,24}$ and inflammation. ${ }^{25,26,27,28}$ Further, it may mention their effectiveness for tolerogenic dendritic cells-based immunotherapy, ${ }^{29}$ and the possibility to use them as nanocarrier ${ }^{30}$.

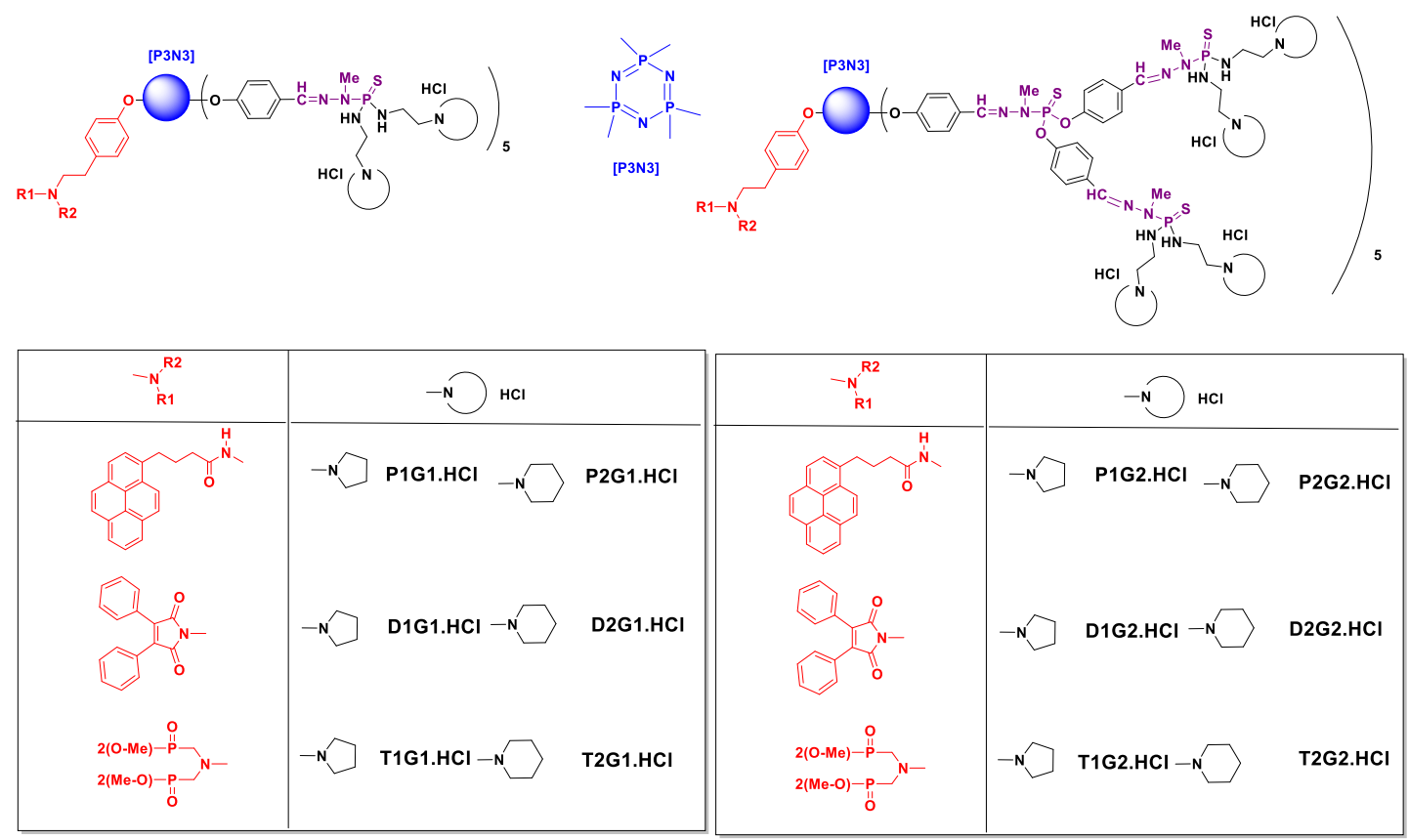

Figure 1. Chemical structure of prepared G1-2 amphiphilic phosphorus dendrons, P1G1.HCI, D1G1.HCI, T1G1.HCI, P2G1.HCI, D2G1.HCI, T2G1.HCl, P1G2.HCI, D1G2.HCI, T1G2.HCI, P2G2.HCI, D2G2.HCI, and T2G2.HCI.

Diverse types of fluorophores have been used as markers of phosphorus dendrimers, which include pyrene, naphthol, anthracene, dansyl, diketone, phthalocyanine, maleimide, julolidine, rhodamine, fluorescein or fluorene 
derivatives. ${ }^{31}$ Fluorescent groups can be introduced onto the surface of dendrimers, linked to the core of dendrimers or attached to branches of dendritic structures. ${ }^{32}$

The first aim of this original study was to prepare original dendron-based micelles with three series of amphiphilic fluorescent cationic phosphorus dendrons (generations 1 and 2) as anticancer agents active per se, that contain two types of protonated cyclic amino groups on their surfaces, namely pyrrolidine and piperidine, a hydrophobic chain that supports a fluorophore moiety such as pyrene and maleimide, and a non-fluorescent azabisdimethyl phosphonate group. In total, twelve macromolecules were prepared and fully characterized (Figure 1). Then, the capacity of amphiphilic dendrons to aggregate in solution, which was mediated by hydrophilic / hydrophobic interactions, to form polymeric micelles was characterized. Lastly, we evaluated the anti-proliferative activities of particles against a panel of tumor cell lines including lung carcinoma A549, breast carcinoma MCF7, breast carcinoma metastasis MDA-MB-231, prostate carcinoma PC3, brain glioblastoma U87-MG, acute promyelocytic leukemia HL60, chronic myelogenous leukemia K562, myelogenous leukemia K562R (imatinib resistant), and colorectal carcinoma HCT116. This research is within our global research goal to develop novel nanotechnology-derived materials for the delivery of drugs, genes, and imaging molecules in the oncology domain.

\section{Results and Discussion}

\section{Synthesis of amphiphilic phosphorus dendrons}

All the reactions were monitored via ${ }^{31} \mathrm{P}$ NMR, and compounds were characterized using ${ }^{31} \mathrm{P}-\mathrm{NMR},{ }^{1} \mathrm{H}-\mathrm{NMR},{ }^{13} \mathrm{C}-\mathrm{NMR}$, and elemental analysis. UV-vis spectroscopic analysis was used to assess fluorescent macromolecules. Purification of the reaction mixture was performed using silica column chromatography. Phosphorus dendrimers are frequently synthesized using a method based on a divergent strategy that consists of the iteration of two key steps using commercially available hexachlorocyclotriphosphazene $\left(\mathrm{P}_{3} \mathrm{~N}_{3} \mathrm{Cl}_{6}\right)(\mathbf{1})$ as a core. ${ }^{33}$ Fluorescent amphiphilic phosphorus dendrons, were formed with an asymmetric structure containing one hydrophobic fluorophore directly grafted to this core on one side, and five other groups that facilitated the development of dendron structures of generations 1 and 2 on the other side. As shown in Scheme 1, the starting point for the synthesis of fluorescent phosphorus dendrons was the preparation of the building block (3) obtained from (1), which involved grafting of five 4-hydroxybenzaldehyde (2). Then, the remaining P-Cl bond was modified via a substitution reaction to incorporate a fluorescent group, either phenol $\mathbf{4 a}$ or $\mathbf{4 b}$, or a non-fluorescent group, phenol 4c. For this purpose, two fluorescent groups including 4-(1-pyrenyl) butyric acid (P) and 2,3-diphenyl maleic anhydride (D) as well as non-fluorescent azabisdimethylphosphonate (T) were prepared and reacted with tyramine to produce a functionalized pyrene monomer (4a), a maleimide functionalized monomer (4b) and an azabisdimethylphosphonate monomer (4c). Then, substituted phenols $4 a-c$ were grafted on the core via nucleophilic substitution of the chlorine atom within THF or acetone solvent in the presence of cesium carbonate to produce 5a-c (83$85 \%$ yield). The next step involved a condensation of monomethylhydrazinodichlorothiophosphate (6) to five aldehyde end groups (compounds $5 \mathrm{a}-\mathrm{c}$ ) in chloroform in the presence of sodium sulfate at $0^{\circ} \mathrm{C}$, which produced dendrons $7 \mathrm{a}-\mathrm{c}$ with yields of $90 \%(7 \mathbf{a}), 91 \%(\mathbf{7 b})$ and $84 \%(7 \mathbf{c})$, respectively. The formation of amphiphilic dendrons requires two additional steps. First, nucleophilic substitution of $-\mathrm{P}(\mathrm{S}) \mathrm{Cl}_{2}$ end groups of $7 \mathrm{a}-\mathrm{c}$ with 2-(pyrrolidin-1-yl)ethan-1-amine (8a) or 2- 
(piperidin-1-yl)ethan-1-amine (8b) give rise to generation 1 phosphorus dendrons including P1G1, D1G1, T1G1, P2G1, D2G1, and T2G1 at yields of $80-90 \%$. These reactions occurred using ten equivalents of $8 \mathbf{a}$ or $\mathbf{8 b}$ in $\mathrm{THF}$ at $0^{\circ} \mathrm{C}$ in the presence of sodium sulfate and DIPEA ( $N, N$-diisopropylethylamine).

The final step of the reactions involved protonation of P1G1, D1G1, T1G1, P2G1, D2G1, and T2G1 through addition of ten equivalents of hydrogen chloride in THF at $0{ }^{\circ} \mathrm{C}$ to produce P1G1.HCl, D1G1.HCI, T1G1.HCI, P2G1.HCl, D2G1.HCl, and T2G1.HCl at yields > 80\%. As depicted in the Scheme 1, a similar strategy was used to prepare phosphorus dendrons of generation 2. The phosphorus dendrons of generation 2 were prepared from dendrons $7 \mathrm{a}-\mathbf{c}$, which were reacted with 10 equivalents of 4-hydroxy-benzaldehyde (2) in THF in the presence of cesium carbonate. This produced dendrons $9 \mathrm{a}-\mathrm{c}$ at yields of $86-92 \%$. Subsequently, condensation of the aldehyde end groups of $9 \mathrm{a}-\mathrm{c}$ with 6, and subsequent addition of nanoparticles $\mathbf{7 a}, \mathbf{7 b}$ or $\mathbf{7 c}$ (20 eq.) in the presence of sodium sulfate and DIPEA produced dendrons P1G2, D1G2, T1G2, P2G2, D2G2, and T2G2. These compounds were protonated with hydrogen chloride in THF at $0{ }^{\circ} \mathrm{C}$ to afford P1G2.HCI, D1G2.HCl, T1G2.HCI, P2G2.HCI, D2G2.HCl, and T2G2.HCl at generally good yields (> $50 \%$ for all three steps of synthesis).

\section{Physical properties of original amphiphilic phosphorus dendron-based micelles}

Twelve different amphiphilic phosphorus dendrons were produced throughout generations 1 and 2, eight of which contained a fluorescent group (P1G1.HCl, D1G1.HCI, P2G1.HCI D2G1.HCI, P1G2.HCl, D1G2.HCI, P2G2.HCl and D2G2.HCI). Next, we aimed to investigate the physical properties of the compounds by assessing their UV-vis absorption and fluorescent emission properties. In addition, critical polymeric micelle concentrations, hydrodynamic size, zeta potential and transmission electron microscopy data were also assessed. Collectively, these data confirmed the feasibility of developing novel nanotechnology using phosphorus dendrons in general. 


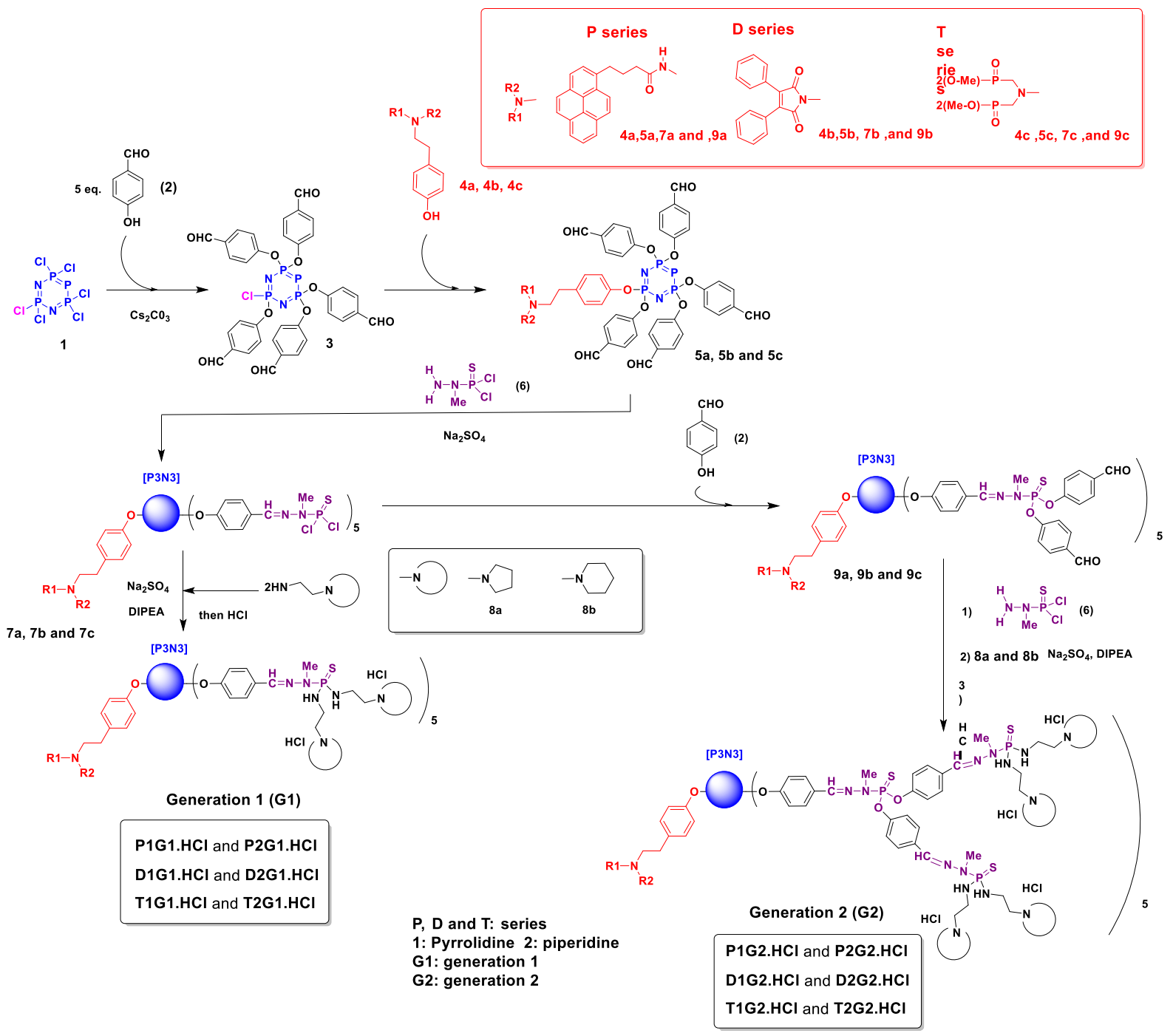

Scheme 1. Synthetic pathways of P1G1.HCI, D1G1.HCI, T1G1.HCl, P2G1.HCl, D2G1.HCl, T2G1.HCl, P1G2.HCl, D1G2.HCl, T1G2.HCI, P2G2.HCI, D2G2.HCI, and T2G2.HCl.

Further, development of a new generation of polymer therapeutics that function via the spontaneous formation of micelles, in particular, is based on self-assembly principles. The optical properties of these amphiphilic fluorescent phosphorus dendrons provided information needed to develop fluorescence imaging applications such as those used in the theranostic field. The 4-(1-pyrenyl) butyric amide series (named P), P1G1.HCI, P2G1.HCI, P1G2.HCI, and P2G2.HCl had high fluorescence quantum yields. In the Figure 2, UV-vis absorption spectra reveal characteristic bands produced via assessment of the pyrene monomer at $244,329,346$ and $376 \mathrm{~nm}$, in addition to a broad band around $278 \mathrm{~nm}$, which corresponds to aromatic units of phosphorus dendrons. 
P1G1.HC

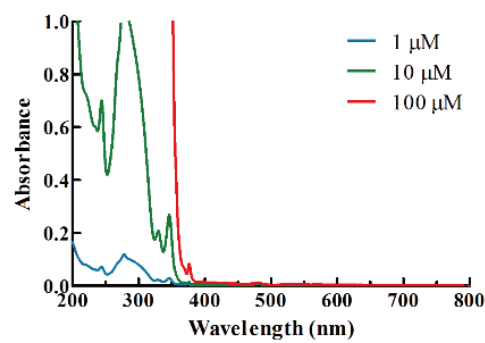

P1G2.HCl

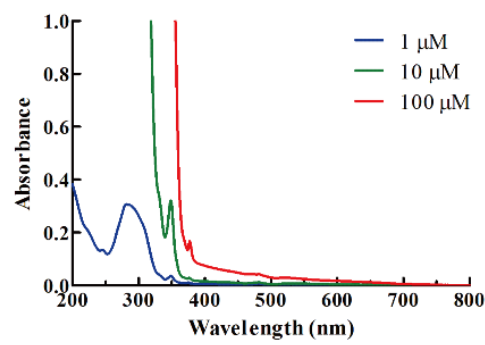

P2G1.HCl

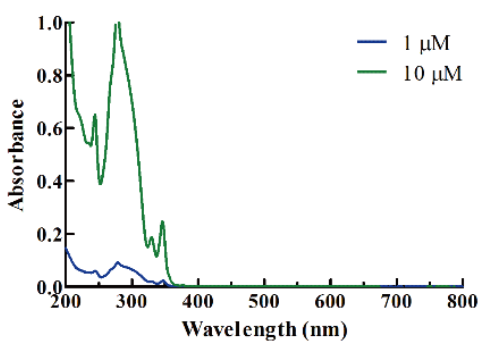

P2G2.HCI

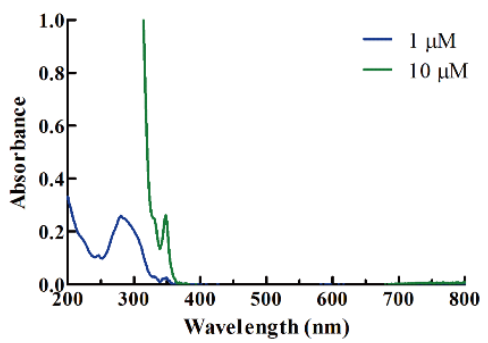

Figure 2. UV-vis absorption spectra of amphiphilic pyrene dendrons dissolved in water.

As shown in Figure 3, the fluorescence spectra of amphiphilic pyrene dendrons reveal excitation bands at 330 and $345 \mathrm{~nm}$, which is consistent with characteristic luminescence peaks typically observed for pyrene monomers. Fluorescence after excitation at $345 \mathrm{~nm}$ produced emission bands at 379,399 and $420 \mathrm{~nm}$. No emission band was observed between 450 and $600 \mathrm{~nm}$, which corresponds to emission patterns of pyrene excimers. The above results indicate that the grafting of pyrene to phosphorus dendrons does not seem to alter the fluorescent properties of pyrene. Also, the formation of pyrene dimers in the excited state, should greatly modify fluorescent wavelength as previously observed, ${ }^{34}$ but is not observed in Figure 3.

P1G1.HCl

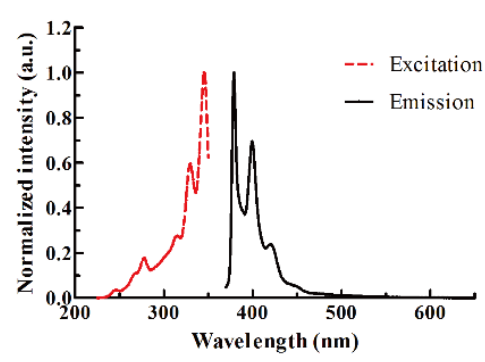

P1G2.HCl

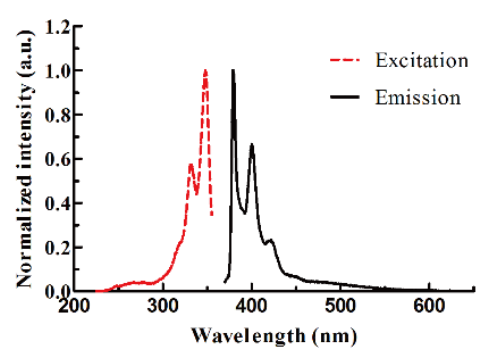

P2G1.HCI

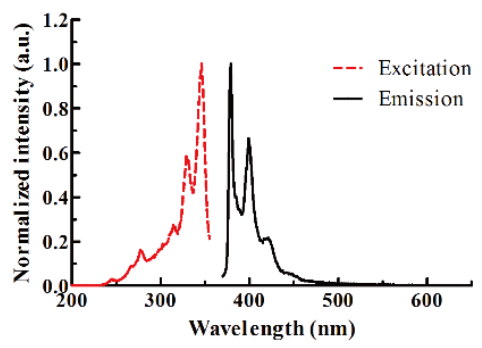

P2G2.HCI

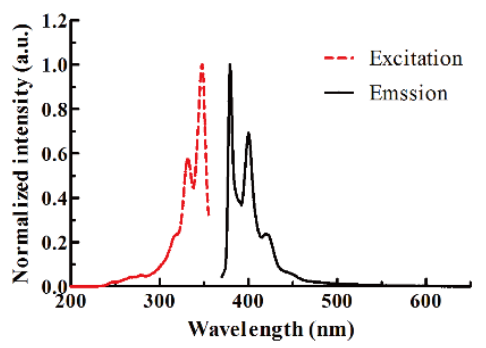

Figure 3. Excitation and emission spectra of amphiphilic pyrene dendrons dissolved in water 
Similar to amphiphilic pyrene dendrons, fluorescent properties of maleimide (named D) were not affected by grafting maleimide to phosphorus dendrons. The UV-vis absorption spectra of amphiphilic maleimide dendrons (macromolecules D1G1.HCI, D2G1.HCI, D1G2.HCI and D2G2.HCl) are shown in Figure S1 (supplementary information). Two broad bands were observed, one near $375 \mathrm{~nm}$, arising from the aromatic groups of maleimide, and another around $284 \mathrm{~nm}$, which corresponded to phosphorus dendrons. Excitation bands around 380-390 nm and the emission bands around 520-540 $\mathrm{nm}$ was present in the fluorescence spectra of amphiphilic maleimide dendrons (Figure S2, supplementary information). Characterization of the amphiphilic azabisdimethylphosphonate dendron (macromolecule T1G1.HCI) that lacked a hydrophobic fluorescent group was also examined for comparison. The UV-vis absorption spectrum of the dendron displayed a broad band around $283 \mathrm{~nm}$ which corresponded to aromatic groups of phosphorus dendron (Figure S3, supplementary information).

Self-assembled micelles are created from amphiphilic compounds that spontaneously form nanosized aggregates after individual surfactants are directly dissolved in aqueous solution above a threshold concentration (critical micelle concentration or $\mathrm{CMC}$ ). ${ }^{35} \mathrm{~A}$ similar phenomenon can be expected with amphiphilic dendrons (Figure 4).

Free-drug dendron micelles
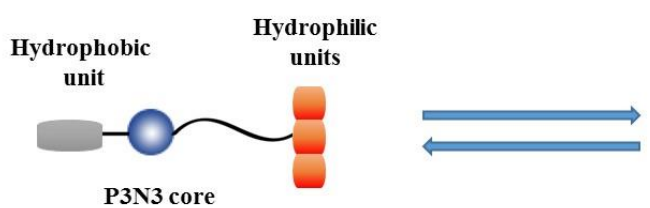

P3N3 core

Amphiphilic dendrons
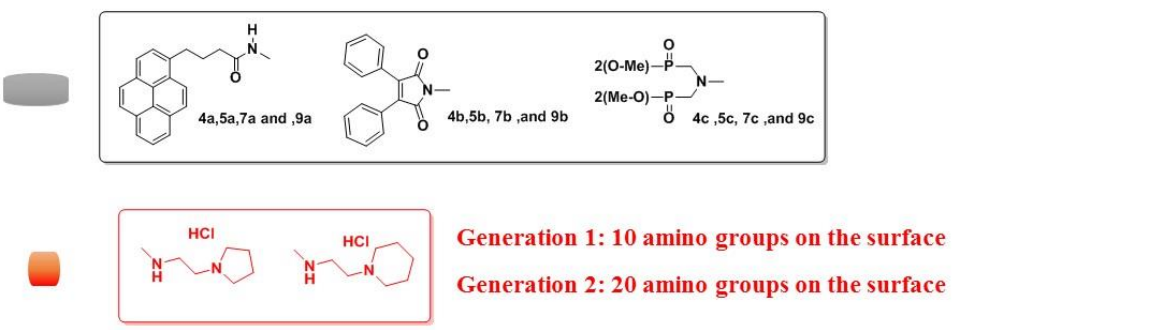

Figure 4. Self-assembly of amphiphilic dendrons to form nanostructured aggregates (micelles).

Table 1. Critical micelle concentrations (CMC) of amphiphilic phosphorus dendrons in water.

\begin{tabular}{lllr}
\hline \multicolumn{1}{c|}{$\mathrm{CMC}(\mu \mathrm{M})$} & Pyrene series $(\mathbf{P})$ & Maleimide series (D) & $\begin{array}{c}\text { Azabisdimethyl } \\
\text { phosphonate series (T) }\end{array}$ \\
\hline Generation 1 with pyrrolidinium & $3.56(\mathbf{P 1 G 1 . H C l )}$ & 76.9 (D1G1.HCl) & $153.7(\mathbf{T 1 G 1 . H C l )}$ \\
\hline
\end{tabular}




\begin{tabular}{|c|c|c|c|}
\hline $\begin{array}{l}\text { Generation } 1 \text { with piperidinium } \\
\text { end groups }\end{array}$ & 2.96 (P2G1.HCI) & 73.3 (D2G1.HCI) & 109 (T2G1.HCI) \\
\hline $\begin{array}{l}\text { Generation } 2 \text { with pyrrolidinium } \\
\text { end groups }\end{array}$ & 1.42 (P1G2.HCI) & 35.3 (D1G2.HCI) & 60 (T1G2.HCI) \\
\hline $\begin{array}{l}\text { Generation } 2 \text { with piperidinium } \\
\text { end groups }\end{array}$ & 2.31 (P2G2.HCI) & 26.3 (D2G2.HCI) & $45.5($ T2G2.HCl) \\
\hline
\end{tabular}

As shown in Table 1, CMC values in water ${ }^{36}$ were based on the capacities of amphiphilic pyrene dendron $\mathbf{P}$ series (P1G1.HCl, P2G1.HCl, P1G2.HCl, and P2G2.HCl), amphiphilic maleimide dendron D series (D1G1.HCI, D2G1.HCI, D1G2.HCl and D2G2.HCl) and azabisdimethylphosphonate dendron T series (T1G1.HCl, T2G1.HCI, T1G2.HCl, and T2G2.HCI) to self-assemble. Values were determined using pyrene as a fluorescent probe for $\mathbf{D}$ and $\mathbf{T}$ series. Amphiphilic pyrene dendron series $(\mathbf{P})$ CMCs were determined via an assessment of their auto-fluorescence spectra. ${ }^{37} \mathrm{CMC}$ was determined by evaluating the ratio of fluorescence intensities measured at emission wavelengths of $373 \mathrm{~nm}\left(\mathrm{I}_{373}\right)$ and 393 $\mathrm{nm}\left(\mathrm{I}_{393}\right)$ for pyrene fluorescence bands at room temperature against the dendron concentration. $I_{373} / I_{393}$ ratio decreases when hydrophobic domains are present in solution since amphiphilic phosphorus dendrons self-assemble into micelles. All tested amphiphilic maleimide phosphorus dendrons (compounds D1G1.HCI, D2G1.HCI, D1G2.HCI, and D2G2.HCI) self-assembled, since decreased $\mathrm{I}_{373} / \mathrm{I}_{393}$ ratios were observed, and CMC values were determined to be $76.9,73.3,35.3$ and $26.3 \mu \mathrm{M}$ respectively (Table 1). Low CMC values, such as those observed here, indicate that amphiphilic dendron micelles remain self-assembled under highly diluted conditions. In addition, our results demonstrated that amphiphilic maleimide dendrons of generation 2 have lower CMC values than their counterparts from generation 1 (D1G1.HCl > D2G1.HCl > D1G2.HCl > D2G2.HCI). Within each generation, the size and the protonation of the cyclic amino ring on the surface also affected steric hindrance (pyrrolidine < piperidine ring). As depicted in Table 1, determined CMC values of azabisdimethylphosphonate dendron (T) micelles followed similar trends as were described for maleimide dendrons (D) previously, in which increased generation number corresponded to decreased CMC value. The higher values of CMC observed with the $\mathbf{T}$ series might be explained by the possible protonation of the nitrogen of the azabisphosphonate units which decrease the hydrophobicity of the core, as a consequence formation of micelles is less favorable. Similar to amphiphilic dendrons $\mathbf{D}$ and $\mathbf{T}$, amphiphilic pyrene dendrons of series $(\mathbf{P})$ of generation 2 had lower CMC values than those of previous generations. Interestingly, the $I_{373} / I_{393}$ ratios decreased within the same generation, for $\mathbf{D}$ and $\mathbf{T}$ series dendrons relative to $\mathbf{P}$ series dendrons (e.g. T1G1. HCl > D1G1. HCl >> P1G1. HCl, T2G2. HCl > D2G2. HCl >> P2G2. HCl), which indicates that quenching effects occur in pyrene series dendrons. Interestingly, as shown in Figure 5, negligible differences between each micelle formed at different concentrations greater than CMC for the pyrene series were observed. This indicated that unimolecular micelles were formed and remained unaltered regardless of dilution (above the $\mathrm{CMC}$ ), which is an attribute that has the potential to be highly useful for in vivo applications. 
P1G1.HCl

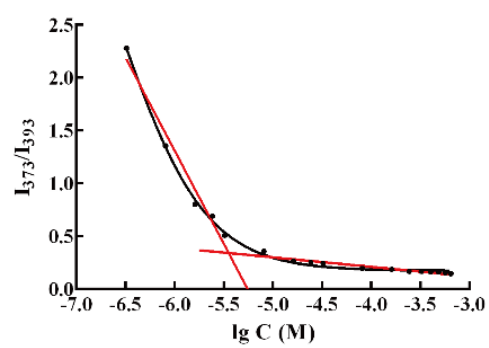

P1G2.HCl

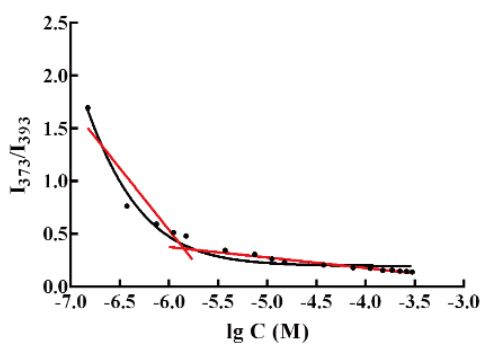

P2G1.HCl

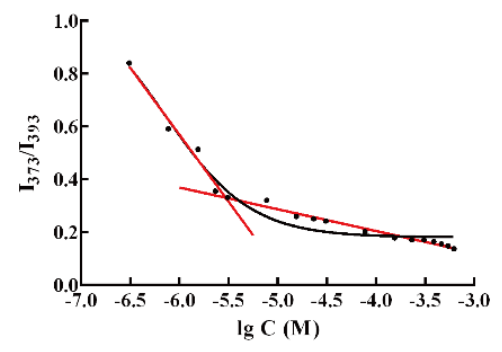

P2G2.HCl

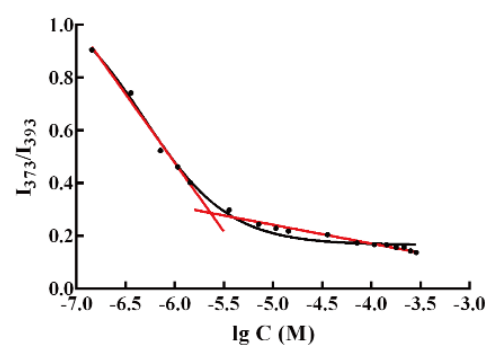

Figure 5 CMC evaluation in the pyrene dendron series. Intensity ratios (I373/I393) as function of logarithm of amphiphilic pyrene dendron concentrations.

In nanomedicine, hydrodynamic size and zeta potential are important general features of nanoparticles, particularly of micelles. These factors affect micelle cytotoxicity, intracellular uptake profiles, their capacities to release drugs to specific tissues, and pharmacokinetic (PK) / pharmacodynamics (PD) profiles. ${ }^{38}$ Dynamic light scattering (DLS) and zeta potential measurements were used to determine the hydrodynamic size of amphiphilic dendron micelles formed from series ( $\mathbf{P}, \mathbf{D}$ and $\mathbf{T})$ dendrons. An outstanding examination by Haag et al. ${ }^{39}$ revealed the relationship between the area under the curve (AUC) pharmacokinetic parameter and hepatic and renal clearance. Further, the researchers assessed the physicochemical properties (size and charge) of dendrimers. Neutral, amphiphilic, weak anionic and weak hydrophobic dendrimers displayed high plasma residence times and long half-lives, which corresponded to low clearance levels. ${ }^{40}$ A recent review by Mignani, Rodrigues, Shi and Majoral et al. assessed the key in-vivo factors important for the development of dendrimers for nanomedical applications. ${ }^{41}$

As shown in Figure 6 (1) and Figure S6, the sizes of amphiphilic dendron micelles ranged from 200 to $500 \mathrm{~nm}$, and increased with generation number. Micelles formed from amphiphilic dendrons containing protonated pyrrolidinium end groups were a bit larger than those formed from amphiphilic dendrons with protonated piperidinium groups of the same generation (micelles of P1G1.HCl > P2G1.HCl, D1G1.HCl > D2G1.HCl, D1G2.HCl > D2G2.HCl, and T1G1.HCl > T2G1.HCl). This trend was not observed when the micelle size of T1G2.HCl was compared with that of T2G2.HCI. Polydispersity index (PDI) values of the twelve amphiphilic dendron micelles were assessed to range from 0.3 to 0.5 , indicating that the mean particle size and homogeneity evaluated were valid (Table S1, supplementary information). To explore the stability of the T2G2. $\mathbf{H C l}$ micelles in both PBS and cell culture medium, their hydrodynamic sizes in both PBS and cell culture medium at $37^{\circ} \mathrm{C}$ were monitored by DLS at different time points. As shown in Figure S7, the hydrodynamic size of T2G2 micelles remains constant for different time periods in both media, indicating their good colloidal stability. 
The slightly larger hydrodynamic size in cell culture medium than that in PBS could be due to the slight protein adsorption, resulting in the formation of protein corona on the surface of the micelles.

Furthermore, the positive surface potential of amphiphilic dendron micelles is an important parameter, which affects their intracellular uptake efficiency and toxicity. The Figure 6 (2) shows the zeta potentials of all the twelve amphiphilic dendron micelles prepared. Zeta potentials determined for amphiphilic pyrene, amphiphilic maleimide, and amphiphilic azabisdimethylphosphonate dendron micelles were determined to be approximately 20,40 , and $36 \mathrm{mV}$, respectively.
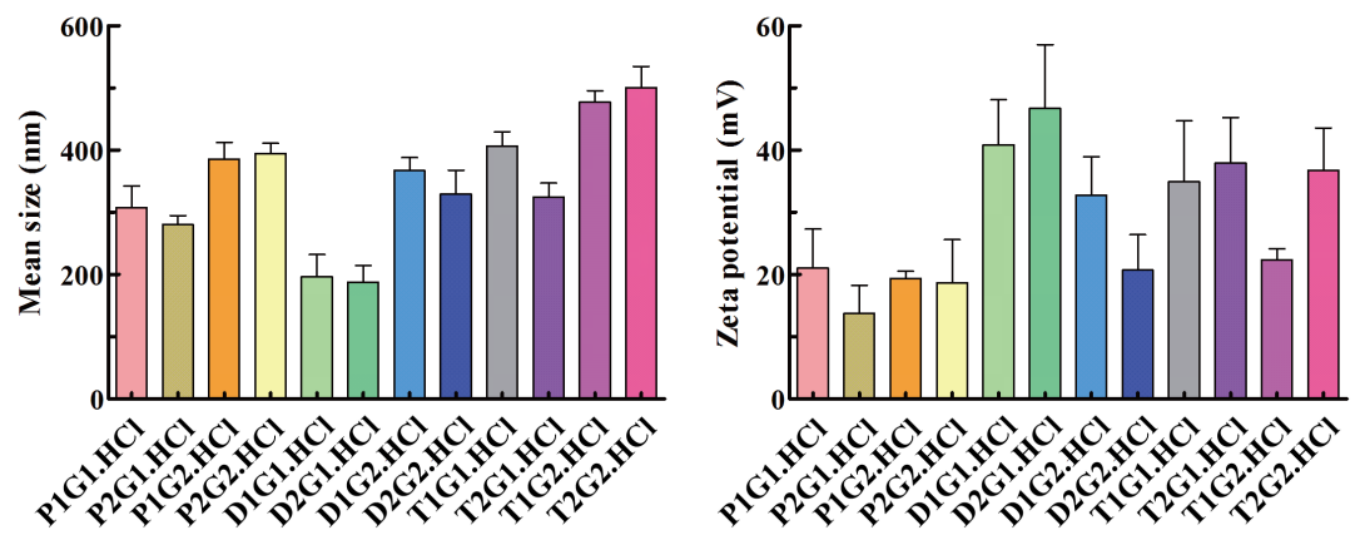

Figure 6. Mean particle size (left) and the zeta potential (right) of the amphiphilic dendron micelles (mean $\pm S D, n=3$ ).

Next, we aimed to assess the morphology and size of aggregates of amphiphilic fluorescent phosphorus dendron micelles using transmission electron microscopy (TEM). P1G1.HCI, P2G1.HCI, P2G2.HCl and D2G1.HCl were selected as representative amphiphilic dendrons based on their fluorescent moieties, terminal amine groups, and variability observed between generations. As shown in Figure 7, all four amphiphilic phosphorus dendrons assessed have shapes that are nearly spherical and the aggregated particles should be ascribed to the drying effect of TEM sample preparation.

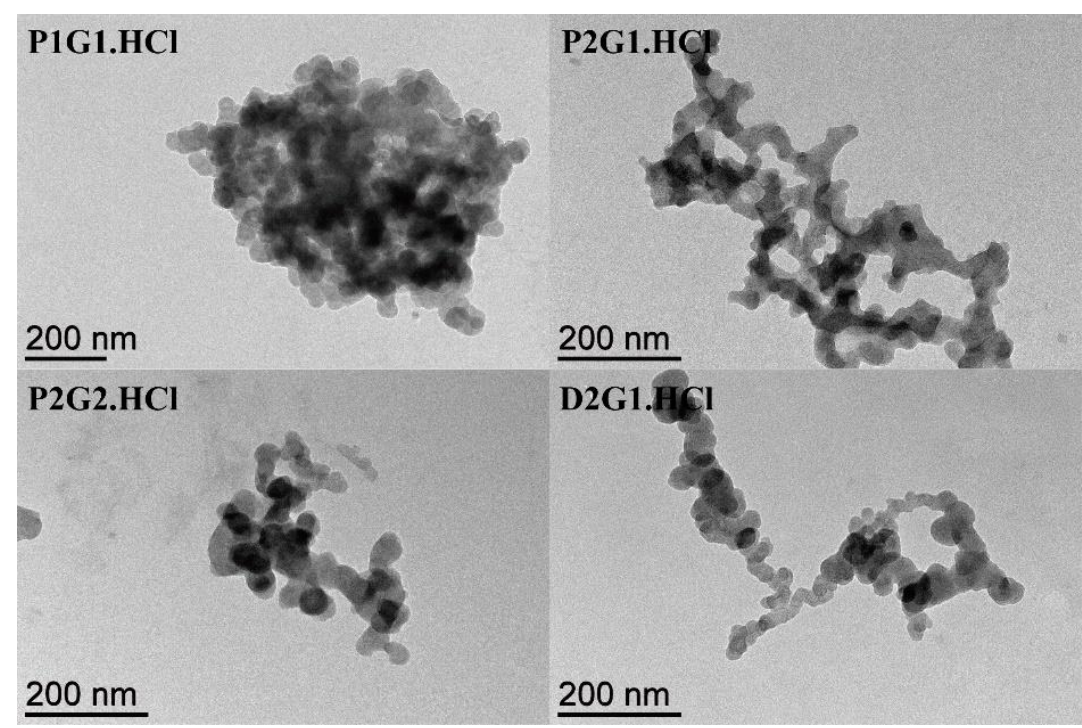

Figure 7. TEM morphology of the amphiphilic fluorescent phosphorus dendron-based micelles. 
The mean diameters determined using TEM (see supplementary information, Figure S8) were approximately 30-40 $\mathrm{nm}$ for all micelles measured, and all were of uniform size distribution. TEM analysis revealed that the mean size of P1G1.HCI, P2G1.HCI, P2G2.HCI and D2G1.HCI micelles was 40, 28, 30 and $31 \mathrm{~nm}$, respectively. Size determined via TEM was smaller than that determined via DLS (vide supra). Such a discrepancy can be reasonably expected as a result of differences between the hydrodynamic diameters of micelles in solution versus the sizes of dry/dehydrated micelles.

\section{Antiproliferative activities of amphiphilic phosphorus dendron-based micelles}

Finally, the cytotoxicity of P1G1.HCl, D1G1.HCl, T1G1.HCl, P2G1.HCl, D2G1.HCl, T2G1.HCI, P1G2.HCI, D1G2.HCI, T1G2.HCI, P2G2.HCI, D2G2.HCl, and T2G2.HCl was assessed using a (3-(4,5-dimethylthiazol-2-yl)-5-(3carboxymethoxyphenyl)-2-(4-sulfophenyl)-2H-tetrazolium) cell viability assay that measured their effects on a panel of tumor cell lines including lung carcinoma A549, breast carcinoma MCF7, breast carcinoma metastasis MDA-MB-231, prostate carcinoma PC3, brain glioblastoma U87-MG, acute promyelocytic leukemia HL60, chronic myelogenous leukemia K562, myelogenous leukemia K562R (doxorubicin resistant due to high P-glycoprotein (PGP) expression), and colorectal carcinoma HCT116. The diploid human fibroblast cell culture line, MRC5, was used to perform safety profiles of compounds. $\mathrm{IC}_{50} \mathrm{~S}$ values ranged between $\sim 0.2$ and $50 \mu \mathrm{M}$, and displayed great heterogeneity between the antiproliferative activities of compounds (see supplementary information). Taken together, antiproliferative activities of compounds against the HCT 116 cell line shown in the Table 2 reveal that dendrons (series $\mathbf{P}, \mathbf{D}$ and $\mathbf{T}$ ) containing piperidinium terminal groups (compounds P2G1.HCI, P2G2.HCI, D2G1.HCI, D2G2.HCI and T2G2.HCI) displayed higher cytotoxicity $\left(\mathrm{IC}_{50} \mathrm{~S}\right.$ 0.2-1.3 $\mu \mathrm{M}$ ) than corresponding dendrons containing pyrrolidinium terminal groups (compounds P1G1.HCl, P1G2.HCI, D1G1.HCI, D1G2.HCI, T1G1.HCl and T1G2.HCI, IC 50 S 0.6-6 $\mu \mathrm{M}, \mathrm{IC}_{50}$ of T1G1.HCl $\left.52 \mu \mathrm{M}\right)$ created within the same generation.

Cytotoxicity increased as generation increased. Compounds of generation 2 were more cytotoxic than those of generation 1, and compounds containing terminal piperidinium groups were particularly effective. This trend was not generally applicable for all cell lines. For instance, against the K562 cell line, the most cytotoxic compounds were those of generation 1. As such, the most potently cytotoxic dendrons were P2G1.HCl and D2G1.HCI $\left(\mathrm{IC}_{50} \mathrm{~S} \sim 0.4-1 \mu \mathrm{M}\right)$ of series P and D, but not series T. Concerning HL60 cell lines, no clear structure activity relationships were observed, and the most potent antiproliferative compound assessed was P2G1.HCI. Unlike doxorubicin (DOX), amphiphilic phosphorus dendrons displayed cell selectivity.

Table 2. Antiproliferative activities of phosphorus dendron micelles and DOX against HCT116, K562 and HCT116 tumor cell lines $\left(\mathrm{IC}_{50}, \mu \mathrm{M}\right)$.

\begin{tabular}{|l|c|c|c|c|}
\hline \multirow{3}{*}{ Series } & \multirow{2}{*}{ Compounds } & \multicolumn{2}{|c|}{ Antiproliferative activities $I_{50}(\mu \mathrm{M})$} \\
\cline { 3 - 5 } & & HL60 & K562 & HCT116 \\
\hline \multirow{3}{*}{$\mathbf{P}$} & P1G1.HCI & $1.17 \pm 0.13$ & $4.62 \pm 0.16$ & $1.54 \pm 0.12$ \\
\cline { 2 - 5 } & P2G1.HCI & $0.28 \pm 0.06$ & $0.36 \pm 0.02$ & $1.07 \pm 0.05$ \\
\hline
\end{tabular}




\begin{tabular}{|c|c|c|c|c|}
\hline & P1G2.HCl & $1.33 \pm 0.2$ & $1.07 \pm 0.01$ & $1.67 \pm 0.13$ \\
\hline & P2G2.HCl & & $3.32 \pm 0.12$ & $0.19 \pm 0.01$ \\
\hline \multirow{4}{*}{ D } & D1G1.HCl & $2.14 \pm 0.15$ & $2.71 \pm 0.7$ & $5.74 \pm 0.21$ \\
\hline & D2G1.HCI & $1.48 \pm 0.01$ & $0.94 \pm 0.001$ & $1.35 \pm 0.24$ \\
\hline & D1G2.HCl & $4.71 \pm 0.052$ & $1.18 \pm 0.11$ & $5.31 \pm 0.04$ \\
\hline & D2G2.HCl & $6 \pm 0.42$ & $10.3 \pm 0.96$ & $0.41 \pm 0.02$ \\
\hline \multirow{5}{*}{$\mathbf{T}$} & T1G1.HCI & $27.9 \pm 6.77$ & $18.2 \pm 2$ & $52.5 \pm 1.64$ \\
\hline & T2G1.HCI & & $5.12 \pm 0.06$ & $9.6 \pm 3.99$ \\
\hline & T1G2.HCI & $3.49 \pm 0.9$ & $1.34 \pm 0.29$ & $3.6 \pm 0.1$ \\
\hline & T2G2.HCI & $4.1 \pm 0.15$ & $1.1 \pm 0.06$ & $0.86 \pm 0.02$ \\
\hline & DOX & $0.01 \pm 0.001$ & $0.05 \pm 0.003$ & $0.09 \pm 0.003$ \\
\hline
\end{tabular}

Table 3. Antiproliferative activities of T2G2.HCl and DOX against normal L929 cells, A549, MCF7, PC3, U87MG, HL60, HCT116, K562, K562R and MDA-MB6231 tumor cell lines (IC $\left.{ }_{50} \mu \mathrm{M}\right)$.

\begin{tabular}{|l|c|c|l|c|c|}
\hline & T2G2.HCI & DOX & & T2G2.HCI & DOX \\
\hline L929 & $8.75 \pm 0.06$ & $0.51 \pm 0.007$ & HL60 & $4.1 \pm 0.15$ & $0.01 \pm 0.001$ \\
\hline A549 & $0.36 \pm 0.02$ & $0.06 \pm 0.001$ & HCT116 & $0.86 \pm 0.02$ & $0.09 \pm 0.003$ \\
\hline MCF7 & $2.89 \pm 0.17$ & $0.12 \pm 0.009$ & K562 & $1.1 \pm 0.06$ & $0.005 \pm 0.003$ \\
\hline PC3 & $0.56 \pm 0.03$ & $0.10 \pm 0.003$ & K562R & $2.47 \pm 0.07$ & $>50$ \\
\hline U87- & $0.27 \pm 0.03$ & $0.01 \pm 0.002$ & MDA-MB- & $0.31 \pm 0.01$ & $0.03 \pm 0.002$ \\
MG & & & 231 & & \\
\hline HL60 & $4.1 \pm 0.15$ & $0.01 \pm 0.001$ & & & \\
\hline
\end{tabular}

Table 4 Safety indexes of T2G2.HCI and DOX against A549, MCF7, PC3, U87-MG, HL60, HCT116, K562, K562R and MDA-MB6231 tumor cell lines.

\begin{tabular}{|c|c|c|c|c|c|}
\hline & T2G2.HCI & DOX & & T2G2.HCI & \\
\hline A549 & $24.31 \pm 1.18$ & $8.5 \pm 0.03$ & HCT116 & $10.17 \pm 0.17$ & $5.67 \pm 0.11$ \\
\hline MCF7 & $3.03 \pm 0.16$ & $4.25 \pm 0.26$ & K562 & $7.95 \pm 0.38$ & $102 \pm 9.34$ \\
\hline PC3 & $15.63 \pm 0.73$ & $5.1 \pm 1.60$ & K562R & $3.54 \pm 0.07$ & $>0.010$ \\
\hline U87-MG & $32.41 \pm 3.42$ & $51 \pm 9.89$ & MDA-MB-231 & $28.22 \pm 0.71$ & $17 \pm 0.90$ \\
\hline HL60 & $2.02 \pm 0.06$ & $51 \pm 4.44$ & & & \\
\hline
\end{tabular}


Interestingly, T2G2. HCl showed broad antiproliferative activity against a panel of tumor cell lines as shown in Table 3. Indeed, strong antiproliferative activities were observed for T2G2.HCl against aggressive A549 (lung carcinoma), PC3 (prostate carcinoma), U87-MG (acute promyelocytic leukemia) and MDA-MB-231 (breast carcinoma metastasis) cells in which $\mathrm{IC}_{50}$ values produced were less than $0.4 \mu \mathrm{M}$. Importantly, T2G2. HCl effectively inhibited proliferation of MDA-MB$231\left(\mathrm{IC}_{50}=0.31 \mu \mathrm{M}\right)$, which is a highly aggressive, invasive and poorly differentiated triple-negative breast cancer (TNBC) cell line. In TNBC, estrogen and progesterone receptors are negative, and treatment options for TNBC are limited. Surgery and chemotherapy, either individually applied, or used in combination, appear to be the only available means to treat the disease, and prognosis remains poor. ${ }^{42} \mathbf{T 2 G 2 . H C l}$ produced no adequate safety ratio versus the MRC5 cell line, which was derived from normal lung tissue. Very interestingly, T2G2. HCl displayed antiproliferative activity against K562R cell line which is resistant to DOX due to its strong PGP expression $\left(\mathrm{IC}_{50}>50\right.$

$\mu \mathrm{M}$ ). Meanwhile, T2G2.HCI micelles and free DOX showed $I_{50}$ S of $8.75 \pm 0.06$ and $0.51 \pm 0.007 \mu \mathrm{M}$ against L929 cells, respectively (Table 3 and Figure S11). Furthermore, the safety indexes of T2G2.HCI and DOX are shown in Table 4. Obviously, T2G2.HCI micelles are much safer than free DOX when they treat A549, PC3, HCT116 and MDA-MB-231 tumor cell lines. To sum up, the T2G2.HCI micelles has a great potential to be used as a drug-free nanoplatform to cure these tumor cells with a high safety and antiproliferative activity.

\section{Conclusion and perspectives}

In medicine, nanotechnology has sparked rapidly growing interest as a result of its potential to solve important issues related to nanomedicine. Nanoformulations have already been used to improve cancer treatments, and several nanoformulated drugs have been FDA approved. Dendrimers represent a new generation of nanoparticle-based therapy. In this article, we describe the straightforward synthesis, full characterization, and antiproliferative activities of novel amphiphilic fluorescent phosphorus dendron-based micelles active per se (generations one and two). These original macromolecules possess both protonated cyclic amino groups and a hydrophobic (fluorescent) moiety, and have the capacity to aggregate in solution via hydrophilic and hydrophobic interactions, which facilitate the formation of polymeric micelles.

This first exploration fully encourages us to design and develop this type of new micelles with the aim to fully control their dimensions, shape and morphology to get nanodevices for in vitro and in vivo biological applications.

Interestingly, several compounds displayed significative antiproliferative activities against acute promyelocytic leukemia HL60, colorectal carcinoma HCT116, breast carcinoma metastasis MDA-MB-231, lung carcinoma A549 and brain glioblastoma U87-MG. The further studies will be to design phosphorus dendron-based micelles with better safety ratio by increasing the length and the nature (e.g. introduction of double bounds) of the chain between the fluorophore and the scaffold as well the nature of the amino groups on the surface. Numerous studies have been carried out to evaluate the in vitro and in vivo cytotoxicity of dendrimers using different cell lines, concentrations, incubation times, and assay methods. The cytotoxicity is strongly influenced by the nature of the functional groups on the dendrimer surface. In the cationic series, like our series presented in this paper, nitrogen functions with decreased basicity display lower cytotoxicity, 
because of their decreased interactions with cell membranes. ${ }^{41,43}$ Another point, will be to evaluate the mechanism of action. Another point will be to evaluate the mechanism of action of these compounds and to determine in more details if they could induced apoptosis and or necrosis cell death. The presented original strategy regarding the development of amphiphilic fluorescent phosphorus dendron-based micelles constitutes a potentially new approach for treating different types of cancer.

The authors declare no competing financial interest.

\section{Supplementary data:}

The Supporting Information is available free of charge at

https://pubs.acs.org/doi/10.1021/acs.bioconjchem.0c00716.

1. Synthesis and characterization; 2. UV-visible and fluorescence properties;3. Critical micelle concentration determination,4. Hydrodynamic size and zeta potential measurements;5. Transmission electron microscope, 6. Biological properties of original amphiphilic fluorescent phosphorus dendrons,7. References, $, 8 .{ }^{31} \mathrm{P},{ }^{1} \mathrm{H}$, and ${ }^{13} \mathrm{C}$ NMR spectra, 7. Biological tests

\section{AUTHOR INFORMATION}

Corresponding Authors

Serge Mignani - Université Paris Descartes, PRES Sorbonne

Paris Cité, CNRS UMR 860, Laboratoire de Chimie et de

Biochimie Pharmacologiques et Toxicologique, 75006 Paris,

France; CQM - Centro de Química da Madeira, MMRG,

Universidade da Madeira, 9020-105 Funchal, Portugal;

Email: serge.mignani@parisdescartes.fr, serge.mignani@

staff.uma.pt

Xiangyang Shi - College of Chemistry, Chemical Engineering

and Biotechnology, Donghua University, Shanghai 201620,

China; CQM - Centro de Química da Madeira, MMRG,

Universidade da Madeira, 9020-105 Funchal, Portugal;

orcid.org/0000-0001-6785-6645; Email: xshi@

dhu.edu.cn

Jean-Pierre Majoral - Laboratoire de Chimie de Coordination

du CNRS, 31077 Toulouse, Cedex 4, France; LCC-CNRS,

Université de Toulouse, CNRS, 31077 Toulouse, France;

orcid.org/0000-0002-0971-817X; Email: majoral@lcctoulouse.

$\mathrm{Fr}$

\section{Authors}

Jieru Qiu - Laboratoire de Chimie de Coordination du CNRS, 31077 Toulouse, Cedex 4, France; College of Chemistry, Chemical Engineering and Biotechnology, Donghua University, Shanghai 201620, China

Liang Chen - Laboratoire de Chimie de Coordination du CNRS, 31077 Toulouse, Cedex 4, France; College of Chemistry, Chemical Engineering and Biotechnology, Donghua University, Shanghai 201620, China

Mengsi Zhan - College of Chemistry, Chemical Engineering and Biotechnology, Donghua University, Shanghai 201620,

China

Régis Laurent - Laboratoire de Chimie de Coordination du CNRS, 31077 Toulouse, Cedex 4, France; LCC-CNRS, Université de Toulouse, CNRS, 31077 Toulouse, France 
Jérôme Bignon - Institut de Chimie des Substances Naturelles

du CNRS, 91198 Paris, Gif-sur-Yvette Cedex, France

Anne-Marie Caminade - Laboratoire de Chimie de

Coordination du CNRS, 31077 Toulouse, Cedex 4, France;

LCC-CNRS, Université de Toulouse, CNRS, 31077

Toulouse, France; orcid.org/0000-0001-8487-3578

Complete contact information is available at:

https://pubs.acs.org/10.1021/acs.bioconjchem.0c00716

Notes

The authors declare no competing financial interest.

\section{Acknowledgements}

This research has been financially supported by the NSFC-CNRS 2019 collaboration project (21911530230 for X.S. and 199675 for R.L., S.M., A.M.C. and J.P.M.), the PRC program NSCF-CNRS 2019 (A.M.C., J.P.M., S.M. and X.S.), the National Key R\&D Program (2017YFE0196200 for X.S.), and the Sino-French Cai Yuanpei Programme. S.M. and X.S. acknowledge the support of FCT-Fundação para a Ciência e a Tecnologia (Base Fund UIDB/00674/2020 and Programmatic Fund UIDP/00674/2020, Portuguese Government Funds) and ARDITI-Agência Regional para o Desenvolvimento da Investigação Tecnologia e Inovação through the project M1420-01-0145-FEDER-000005-CQM+ (Madeira 14-20 Program). S.M. and J.P.M. acknowledge transnational EuroNanoMed III funded projects including a proper acknowledgement of ERANET EuroNanoMed III and the respective funding partner organizations. J.P.M., A.M.C. and R.L. thanks CNRS (France) for financial support.

\section{References}

1. Kim, H. J.; Kim, A.; Miyata, K.; Kataoka, K., Recent progress in development of siRNA delivery vehicles for cancer therapy. Adv. Drug Delivery Rev. 2016, 104, 61-77.

2. Tran, S.; DeGiovanni, P. J.; Piel, B.; Rai, P., Cancer nanomedicine: A review of recent success in drug delivery. Clin. Transl. Med. 2017, 6, 44.

3. Ulbrich, K.; Hola, K.; Subr, V.; Bakandritsos, A.; Tucek, J.; Zboril, R., Targeted drug delivery with polymers and magnetic nanoparticles: Covalent and noncovalent approaches, release control, and clinical studies. Chem. Rev. 2016, 116 (9), 5338-5431.

4. Cabral, H.; Miyata, K.; Osada, K.; Kataoka, K., Block copolymer micelles in nanomedicine applications. Chem. Rev. 2018, $118(14), 6844-6892$.

5. Varela-Moreira, A.; Shi, Y.; Fens, M. H. A. M.; Lammers, T.; Hennink, W. E.; Schiffelers, R. M., Clinical application of polymeric micelles for the treatment of cancer. Mater. Chem. Front. 2017, 1 (8), 1485-1501.

6. Saif, M. W.; Podoltsev, N. A.; Rubin, M. S.; Figueroa, J. A.; Lee, M. Y.; Kwon, J.; Rowen, E.; Yu, J.; Kerr, R. O., Phase II clinical trial of paclitaxel loaded polymeric micelle in patients with advanced pancreatic cancer. Cancer Invest. 2010,28 (2), 186-194.

7. Kim, Y.; Park, E. J.; Na, D. H., Recent progress in dendrimer-based nanomedicine development. Arch. Pharmacal Res. 2018, 41 (6), 571-582.

8. $\quad$ Esfand, R.; Tomalia, D. A., Poly(amidoamine) (PAMAM) dendrimers: From biomimicry to drug delivery and biomedical applications. Drug Discovery Today 2001, 6 (8), 427-436.

9. De Brabander van den Berg, E. M. M.; Meijer, E. W., Poly(propylene imine) dendrimers: Large-scale synthesis by hetereogeneously catalyzed hydrogenations. Angew. Chem., Int. Ed. Engl. 1993, 32 (9), 1308-1311.

10. Picart, C.; Lavalle, P.; Hubert, P.; Cuisinier, F. J. G.; Decher, G.; Schaaf, P.; Voegel, J. C., Buildup mechanism for poly(L-lysine)/hyaluronic acid films onto a solid surface. Langmuir 2001, 17 (23), 7414-7424.

11. Darrabie, M. D.; Kendall, W. F.; Opara, E. C., Characteristics of poly-L-ornithine-coated alginate microcapsules Biomaterials 2005, 26 (34), 6846-6852.

12. Zhou, L. L.; Roovers, J., Synthesis of novel carbosilane dendritic macromolecules. Macromolecules 1993, 26 (5), 963-

968.

13. Launay, N.; Caminade, A. M.; Lahana, R.; Majoral, J. P., A general synthetic strategy for neutral phosphoruscontaining dendrimers Angew. Chem., Int. Ed. Engl. 1994, 33 (15-16), 1589-1592.

14. Caminade, A. M.; Turrin, C. O.; Laurent, R.; Ouali, A.; Delavaux-Nicot, B., Dendrimers: Towards catalytic, material and biomedical uses. John Wiley \& Sons Ltd.Chichester, UK. 2011.

15. Caminade, A. M.; Turrin, C. O.; Majoral, J. P., Phosphorous dendrimers in biology and nanomedicine: Syntheses, characterization, and properties. Jenny Stanford Publishing 2018. 
16. Mignani, S. M.; El Brahmi, N.; El Kazzouli, S.; Laurent, R.; Ladeira, S.; Caminade, A. M.; Pedziwiatr-Werbicka, E.; Szewczyk, E. M.; Bryszewska, M.; Bousmina, M. M.; Cresteil, T.; Majoral, J. P., Original multivalent gold(III) and dual gold(III)copper(II) conjugated phosphorus dendrimers as potent antitumoral and antimicrobial agents. Mol. Pharmaceutics 2017, 14 (11), 4087-4097.

17. Wasiak, T.; Ionov, M.; Nieznanski, K.; Nieznanska, H.; Klementieva, O.; Granell, M.; Cladera, J.; Majoral, J. P.; Caminade, A. M.; Klajnert, B., Phosphorus dendrimers affect alzheimer's (A 1 1-28) peptide and MAP-tau protein aggregation Mol. Pharmaceutics 2012, 9 (3), 458-469.

18. Solassol, J.; Crozet, C.; Perrier, V.; Leclaire, J.; Beranger, F.; Caminade, A. M.; Meunier, B.; Dormont, D.;

Majoral, J. P.; Lehmann, S., Cationic phosphorus-containing dendrimers reduce prion replication both in cell culture and in mice infected with scrapie. J. Gen. Virol. 2004, 85, 1791-1799.

19. Klajnert, B.; Cortijo-Arellano, M.; Cladera, J.; Majoral, J. P.; Caminade, A. M.; Bryszewska, M., Influence of phosphorus dendrimers on the aggregation of the prion peptide PrP 185-208. Biochem. Biophys. Res. Commun. 2007, 364 (1), 20-25.

20. Ottaviani, M. F.; Mazzeo, R.; Cangiotti, M.; Fiorani, L.; Majoral, J. P.; Caminade, A. M.; Pedziwiatr, E.;

Bryszewska, M.; Klajnert, B., Time evolution of the aggregation process ofpeptides involved in neurodegenerative diseases and preventing aggregationeffect of phosphorus dendrimers studied by EPR. Biomacromolecules 2010, 11 (11), 3014-3021.

21. Wasiak, T.; Marcinkowska, M.; Pieszynski, I.; Zablocka, M.; Caminade, A. M.; Majoral, J. P.; Klajnert-Maculewicz, B., Cationic phosphorus dendrimers and therapy for Alzheimer's disease. New J. Chem. 2015, 39 (6), $4852-4859$.

22. Milowska, K.; Gabryelak, T.; Bryszewska, M.; Caminade, A. M.; Majoral, J. P., Phosphorus-containing dendrimers against a-synuclein fibril formation. Int. J. Biol. Macromol. 2012, 50 (4), 1138-1143.

23. Lazniewska, J.; Milowska, K.; Zablocka, M.; Mignani, S.; Caminade, A. M.; Majoral, J. P.; Bryszewska, M.;

Gabryelak, T., Mechanism of cationic phosphorus dendrimer toxicity against murine neural cell lines. Mol. Pharmaceutics 2013, 10 (9), 3484-3496.

24. Klajnert, B.; Cangiotti, M.; Calici, S.; Majoral, J. P.; Caminade, A. M.; Cladera, J.; Bryszewska, M.; Ottaviani, M. F., EPR study of the interaction between dendrimers and peptides involved in Alzheimer's and prion diseases. . Macromol. Biosci. 2007, 7, 1065-1074.

25. Hayder, M.; Poupot, M.; Baron, M.; Nigon, D.; Turrin, C. O.; Caminade, A. M.; Majoral, J. P.; Eisenberg, R. A.; Fournié, J. J.; Cantagrel, A.; Poupot, R.; Davignon, J. L., A phosphorus-based dendrimer targets inflammation and osteoclastogenesis in experimental arthritis. Sci. Transl. Med. 2011, 3 (81), 81ra35.

26. Blattes, E.; Vercellone, A.; Eutamène, H.; Turrin, C. O.; Théodorou, V.; Majoral, J. P.; Caminade, A. M.; Prandi, J.; Nigou, J.; Puzo, G., Mannodendrimers prevent acute lung inflammation by inhibiting neutrophil recruitment. Proc. Natl. Acad. Sci. 2013, 110 (22), 8795-8800.

27. Vacas-Cordoba, E.; Bastida, H.; Pion, M.; Hameau, A.; Ionov, M.; Bryszewska, M.; Caminade, A. M.; Majoral, J.

P.; Munoz-Fernandez, M. A., HIV-antigens charged on phosphorus dendrimers as tools for tolerogenic dendritic cells-based immunotherapy. Curr. Med. Chem. 2014, 21 (16), 1898-1909.

28. Loup, C.; Zanta, M. A.; Caminade, A. M.; Majoral, J. P.; Meunier, B., Preparation of water-soluble cationic phosphorus-containing dendrimers as DNA transfecting agents. Chem. - Eur. J. 1999, 5 (12), 3644-3650.

29. Wei, T.; Chen, C.; Liu, J.; Liu, C.; Posocco, P.; Liu, X. X.; Cheng, Q.; Huo, S. D.; Liang, Z. C.; Fermeglia, M.;

Pricl, S.; Liang, X. J.; Rocchi, P.; Peng, L., Anticancer drug nanomicelles formed by self-assembling amphiphilic dendrimer to combat cancer drug resistance. Proc. Natl. Acad. Sci. 2015, 112 (10), 2978-2983.

30. $\quad$ Chen, L.; Li, J.; Fan, Y.; Qiu, J.; Cao, L.; Laurent, L.; Mignani, S.; Caminade, A. M.; Majoral, J.-P.; X., S., Revisiting cationic phosphorus dendrimers as a nonviral vector for optimized gene delivery toward cancer therapy applications. Biomacromolecules, 2020, 21, 2502-2511.

31. Qiu, J. R.; Hameau, A.; Shi, X. Y.; Mignani, S.; Majoral, J. P.; Caminade, A. M., Fluorescent phosphorus dendrimers: Towards material and biological applications. ChemPlusChem 2019, 84 (8), 1070-1080.

32. Caminade, A. M.; Hameau, A.; Majoral, J. P., Multicharged and/or water-soluble fluorescent dendrimers: Properties and uses. Chem. Eur. J. 2009, 15 (37), 9270-9285.

33. Lartigue, M. L.; Donnadieu, B.; Galliot, C.; Caminade, A. M.; Majoral, J. P.; Fayet, J. P., Large dipole moments of phosphorus-containing dendrimers. Macromolecules 1997, 30 (23), 7335-7337.

34. Brauge, L.; Caminade, A. M.; Majoral, J. P.; Slomkowski, S.; Wolszczak, M., Segmental mobility in phosphoruscontaining dendrimers. Studies by fluorescent spectroscopy. Macromolecules 2001, 34 (16), 5599-5606.

35. Chakraborty, T.; Chakraborty, I.; Ghosh, S., The methods of determination of critical micellar concentrations of the amphiphilic systems in aqueous medium. Arabian J. Chem. 2011, 4 (3), 265-270.

36. Kalyanasundaram, K.; Thomas, J. K., Environmental effects on vibronic band intensities in pyrene monomer

fluorescence and their application in studies of micellar systems. J. Am. Chem. Soc. 1977, 99 (7), $2039-2044$.

37. Dargel, C.; Geisler, R.; Hannappel, Y.; Kemker, I.; Sewald, N.; Hellweg, T., Self-assembly of the bio-surfactant aescin in solution: A small-angle X-ray scattering and fluorescence study. Colloids and Interfaces 2019, 3 (2), 47-61.

38. He, C. B.; Hu, Y. P.; Yin, L. C.; Tang, C.; Yin, C. H., Effects of particle size and surface charge on cellular uptake and biodistribution of polymeric nanoparticles. Biomaterials 2010, 31 (13), 3657-3666.

39. Khandare, J.; Calderon, M.; Dagia, N. M.; Haag, R., Multifunctional dendritic polymers in nanomedicine: opportunities and challenges. Chem. Soc. Rev. 2012, 41, 2824-2848.

40. Qiao, H. Z.; Li, J.; Wang, Y.; Ping, Q. N.; Wang, G. J.; Gu, X. C., Synthesis and characterization of multi-functional linear-dendritic block copolymer for intracellular delivery of antitumor drugs. Int. J. Pharm. 2013, 452 (1-2), 363-373.

41. Mignani, S.; Rodrigues, J.; Roy, R.; Shi, X. Y.; Cena, V.; El Kazzouli, S.; Majoral, J. P., Exploration of biomedica dendrimer space based on in-vivo physicochemical parameters: Key factor analysis (Part 2). Drug Discovery Today 2019,24 (5), 1184-1192.

42. Malorni, L.; Shetty, P. B.; De Angelis, C.; Hilsenbeck, S.; Rimawi, M. F.; Elledge, R.; Osborne, C. K.; De Placido, S.; Arpino, G., Clinical and biologic features of triple-negative breast cancers in a large cohort of patients with long-term followup. Breast Cancer Res. Treat. 2012, 136 (3), 795-804.

43. Mignani, S.; Rodrigues, J.; Roy, R.; Shi, X.; Ceña, V.; El Kazzouli, S.; Majoral, J.-P., Exploration of biomedical dendrimer space based on in-vitro physicochemical parameters: key factor analysis (Part 1). Drug Discov. Today 2019, 24, 1176-1183. 